\title{
CaReMoOC: Capacity, Reserve, Movement Objectives, and Compensation. A new framework to describe mechanisms of movement limitations, demonstrated for ageing.
}

\footnotetext{
Eline van der Kruk*, Department of Bioengineering, Imperial College London, London, UK - e.van-der-kruk@imperial.ac.uk Imperial College London |Bessemer Building |London SW7 2AZ

Anne K. Silverman, Department of Mechanical Engineering, Colorado School of Mines, Golden, USA - asilverm@mines.edu Louis Koizia, Department of Medicine, Imperial College Healthcare, London UK - I.koizia@nhs.net Peter Reilly, Department of Orthopaedics, Imperial College Healthcare, London UK - p.reilly@imperial.ac.uk Michael Fertleman, Department of Medicine, Imperial College Healthcare, London UK - $\underline{\text { m.fertleman@imperial.ac.uk }}$ Anthony M.J. Bull, Department of Bioengineering, Imperial College London, London, UK - a.bull@imperial.ac.uk

* corresponding author
} 


\begin{abstract}
To prevent, mitigate and treat movement impairments, we need to recognize early signs of decline and understand how to best compensate for limitations. The mechanisms leading to movement impairments are complex, overlapping, and interdependent and the fields of biomechanics, motor control, and physiology must be combined to understand these mechanisms. This article introduces CaReMoOC, a framework incorporating neuromusculoskeletal capacity (accumulation of neuromusculoskeletal resources over the lifespan), reserve (task-specific difference between capacity and task demand), movement objectives (considerations made to plan a movement), and compensation (use of NMSK resources to respond to the task demand). The framework is demonstrated for healthy ageing, providing an overview of age-related capacity decline (neural, skeletal, muscular system) and shifted weighting of movement objectives (energy, pain, stability, speed) relevant for biomechanics and motor control. Two forms of compensation are Compensation for Capacity, when capacity does not meet the task demands, and Compensation for Movement Objectives, when the movement is changed due to for example a fear of falling. Understanding the interrelationships between decline in the variables within capacity and the effect on compensation strategies will provide benefit in preventing mobility impairments and will support clinicians in their rehabilitation practice.
\end{abstract}

Keywords: Ageing, Human Movement, Mobility Impairments, Capacity, Reserve, Compensation, Biomechanics, Geriatrics, Modelling, Rehabilitation 


\section{Background}

Consistently low birth rates and higher life expectancy are transforming society, resulting in the proportional and absolute increase in an ageing population. Rapid ageing is occurring world-wide, so that by 2050 all regions except for Africa will have at least $25 \%$ of their population over 60 years old; the proportion of people aged 80 or over will have tripled by that time (1). With ageing comes a decrease in mobility and so maintaining mobility is critical for elderly people to remain independent, to be able to work, and to enhance social inclusion.

To maintain mobility and prevent mobility impairments, we need to be able to recognize early signs of decline and understand how to compensate for limitations in movement. The fields of biomechanics and motor control seek to understand the mechanisms of age-related mobility decline by understanding forces, deformation, control, and motion of biological systems, while the field of physiology focusses on the biological processes. Daily life activities such as walking, standing up from a chair, or ascending stairs are complex motor tasks. The mechanisms that contribute to mobility impairments in these daily life activities are complex, overlapping, and interdependent. Consequently, the fields of biomechanics, motor control, and physiology must be combined to understand these mechanisms.

Further adding to the challenge of research on the biomechanics of ageing is the lack of general applicable and consensus terminology. Terms such as physiological capacity (2), musculoskeletal reserve (3), and musculoskeletal capacity (4) have been previously introduced, but a general understanding and definition of these terms in the fields of biomechanics and motor control is lacking.

This article introduces a general framework to describe mechanisms of movement limitations providing definitions of neuromusculoskeletal capacity, reserve, movement objectives, and compensation (CaReMoOC from now on). The framework can be generally applied to a variety of pathologies, patient or participant groups, and motor tasks. In this article we demonstrate the framework for ageing and provide a complete overview of the age-related declining factors relevant for biomechanics and motor control. 


\section{CaReMoOC: Capacity, Reserve, Movement Objectives, and Compensation}

\subsection{Neuromusculoskeletal capacity}

Neuromusculoskeletal capacity is defined as the physiological abilities of the neuro-musculoskeletal system. Capacity accumulates due to genetic and/or environmental factors up to a point at which agerelated decline sets in (Figure 1). A higher peak capacity mitigates the effects of decline caused by ageing or age-related diseases and the rate of decline can be adjusted through environmental factors.

Capacity accumulation can be compared to charging a capacitor (Figure 1). Charging a capacitor requires an active source, which is comparable to an active lifestyle. The capacitance value of the capacitor can be compared to genetics. This determines both the time it takes to charge the capacitor and, together with the active source, the maximum capacitor value (Figure 1b). The resistor resembles environmental effects and lifestyle choices such as nutrition, drug and/or alcohol use, and illnesses. The resistor (R1) determines, together with the capacitance, the time it takes to charge the capacitor. The switch resembles the start of decline. When the switch is turned, the capacitor is no longer being charged, but will be discharged. The time it takes to discharge is dependent on the initial value of the capacitor (peak reserve) and the value of the resistor (R2). More resistance to the discharge, will slow down the decline.

Decline of neuromusculoskeletal (NMSK) capacity is a result of structural changes of the neural, muscular, and skeletal (including soft tissues) systems, which are reviewed in detail in Section 3-5. NMSK reserve does not account for changes in the endocrine, immune, cardiovascular, respiratory, renal, or brain systems. The NMSK capacity determines whether an individual can overcome a stressor such as the consequences of a fall and therefore key factors to be considered are peak capacity and slope of decline. 
a. environmental effects, lifestyle (e.g. nutrition, drug use)

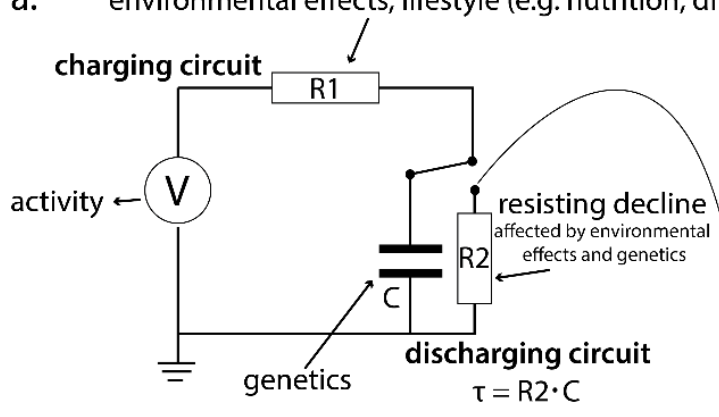

b.
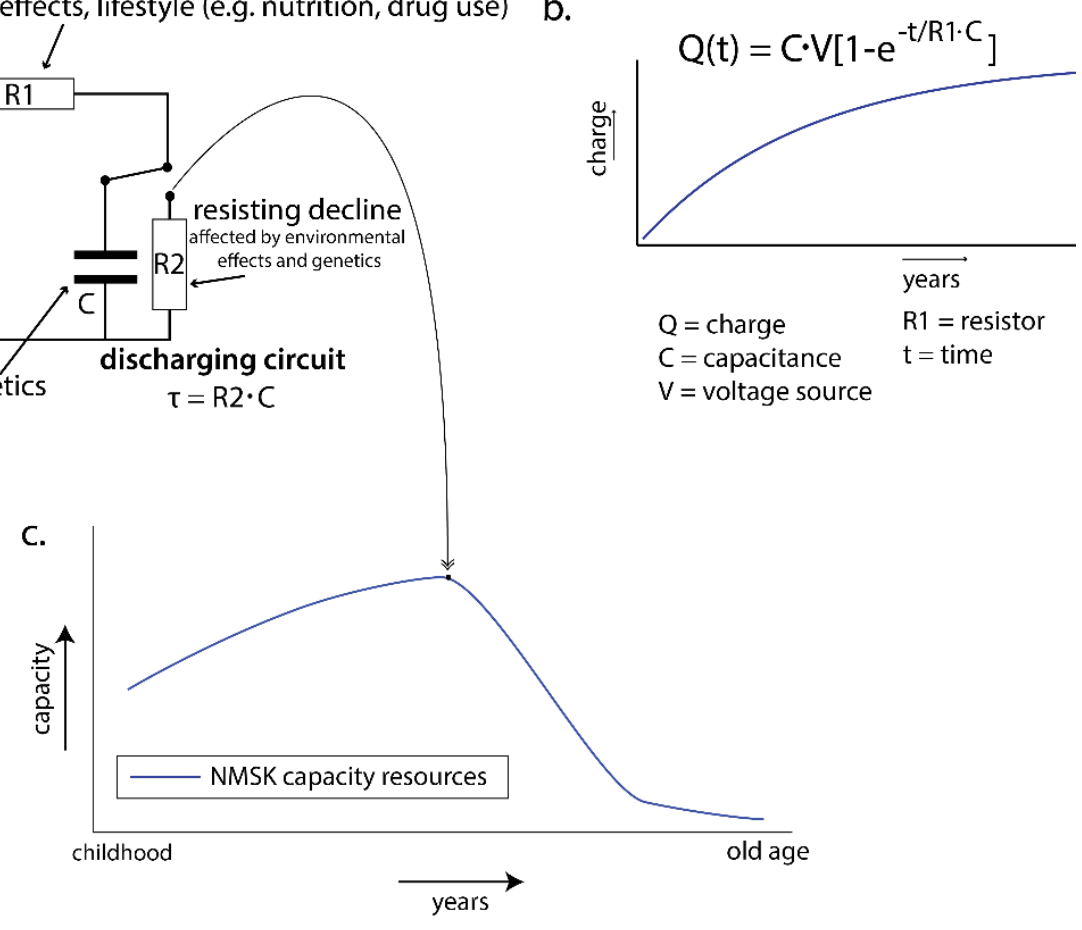

FIGURE 1: Neuromusculoskeletal capacity accumulation compared to charging a capacitor. In the charging mode, the voltage source resembles activity, the capacitor the genetics, and the resistor the environment and lifestyle (e.g. nutrition, drug use) (a). The eventual charge is determined by the capacitor (genetics) and the voltage source (activity), whereas resistor R1 (environment \& lifestyle) and capacitance determine the speed of charging (b). The switch closes and thereby determines the peak capacity (c). Subsequently, the capacitor discharges. The slope of decline ( $\tau=$ time constant of decline) is determined by resistor R2 (environment \& lifestyle) and the capacitance (genetics). 


\subsection{Neuromusculoskeletal reserve}

NMSK reserve is task specific and is the difference between the capacity and the task demands (Figure 2). Reserve describes whether we can execute a task and how much reserve there is left before the onset of task inability. As task requirements vary over the duration of the task, so does reserve, therefore, inability may occur for only a portion of the task but still results in task failure. For example, in standing up, the point of lift off from the chair has the highest task demand and the reserve for this part of the task is therefore smallest. It is likely that this part of the task execution will become impaired first, unless there is room for compensation, described later.

a.

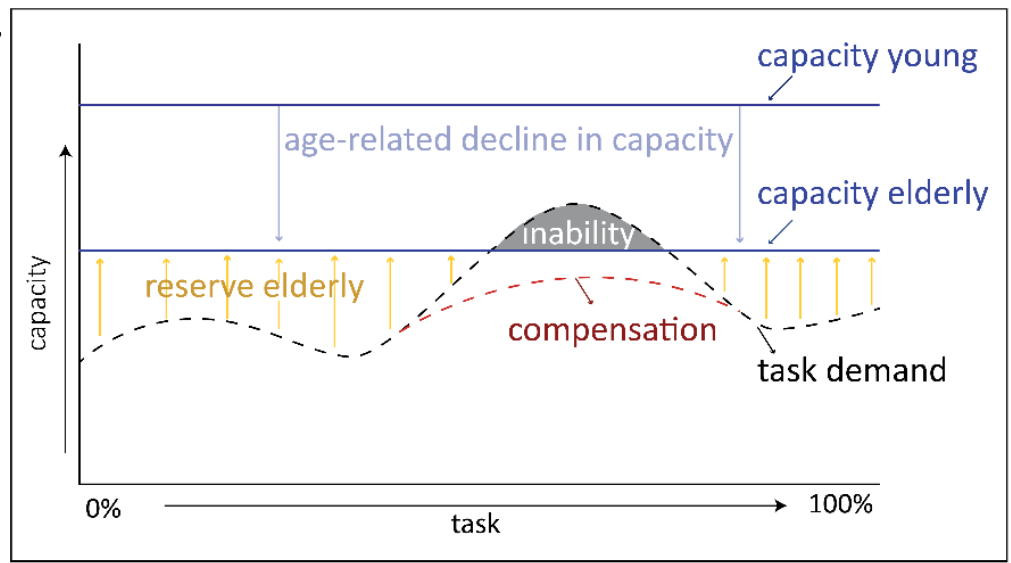

b.
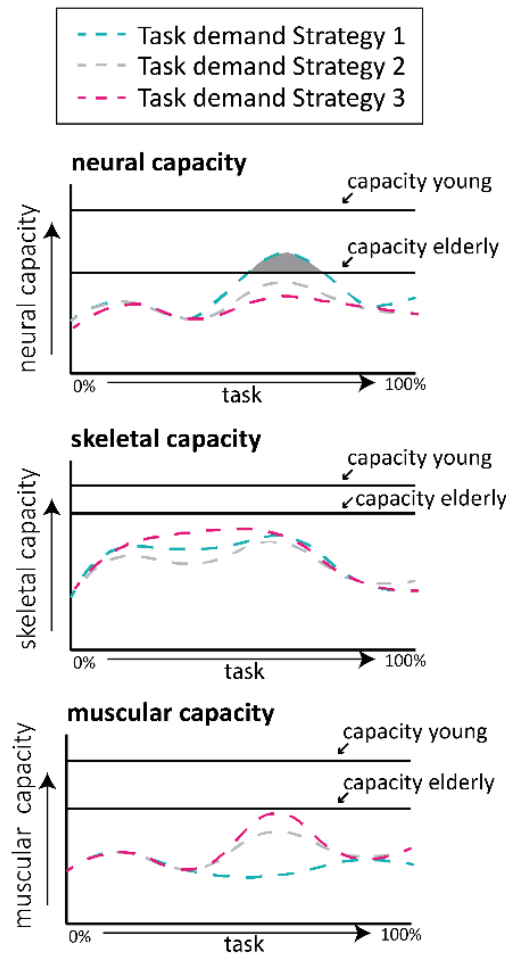

FIGURE 2: a) Task-specific overview of capacity, reserve, and compensation. Capacity is defined as the physiological abilities of the neuromusculoskeletal system, in this case available for this task. Reserve is the difference between the capacity and the task demands. If the reserve cannot meet the task demands, compensation can be applied to adjust the task while achieving the same goal. b) task-specific overview of capacity separated into its main components: neural, skeletal, and muscular capacity. Movement strategies have a different task demand on each of these elements and the systems can compensate for each other. Note that within each of the components there are sub-components for which these same concepts hold. For example, in the muscular capacity we could distinguish between the lower and upper body muscles, muscle groups, or individual muscles where different strategies result in different task demands on each of the sub-components. In this theoretical example, Strategy 1 cannot be executed by an elderly person due to a lack of neural capacity, and Strategy 3 can only just be executed by an elderly person due to zero reserve in muscular capacity. 


\subsection{Movement objectives}

Within the redundancy of capacity and reserve, humans both consciously and unconsciously decide on movement strategies (Figure 3). To reach the movement goal (e.g. standing up), there are several feasible strategies within this capacity to reach this goal each with their own task demands (e.g. standing up with or without using arms). The applied motion strategy of humans considers metabolic energy, speed, safety (e.g. stability margins), and/or pain avoidance, which will be jointly referred to as the movement objectives. Each strategy can attribute a different weighting to these movement objectives, resulting in different movement strategies. Although movement objectives are not age dependent, the relative weighting of these objectives most likely are, thus resulting in different movement strategies at different ages. The movement objectives and their relative weighting are a multi-objective function determining the most effective strategy. Humans make comparative assessments of movement objectives based on the task goal, their capacity, and psychological reasons such as fear of falling, pain, or an unknown environment (5) (Figure 3).

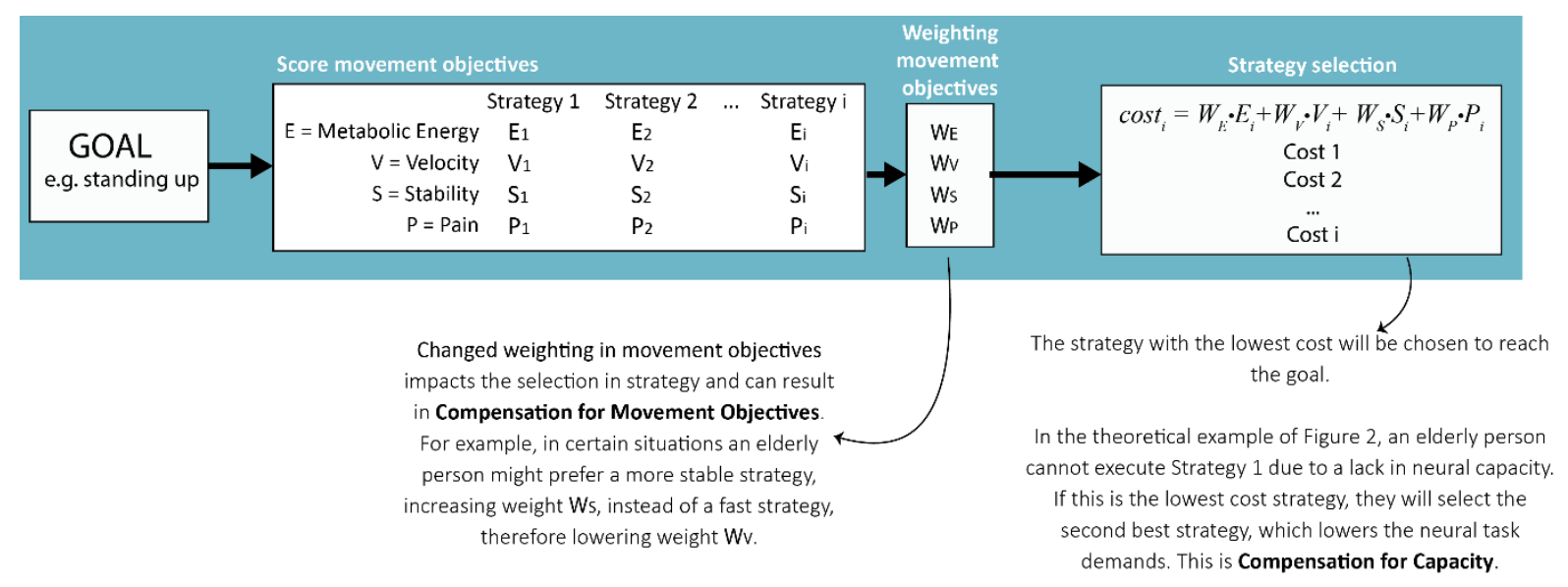

FIGURE 3: Movement Objectives and Compensation strategies. Goal is the aim of the task and has certain inherent constraints, such as gravity, not falling over, or avoiding objects. Within the capacity there are several possible movement strategies, each with their own task demands (Figure 2). These strategies can each be assigned a score on the movement objectives (Energy, Velocity, Stability, and Pain). Combining these scores with the weighting in movement objectives in a multi-objective function results in an overall score. The strategy with the lowest score will be selected. Compensation for capacity occurs when capacity does not meet the task demands of the lowest cost strategy (global minimum), the next best strategy will be selected (local minimum). Compensation for Movement Objectives occurs when, compared to a baseline, the choice in strategy has changed due to changed movement objectives. 


\subsection{Neuromusculoskeletal compensation}

A movement strategy is defined as compensation in relation to a baseline (e.g. previous state or a control group). We distinguish two cases of compensation:

- Compensation for Capacity: relates to task-performance enhancing recruitment of NMSK recourses in response to a relatively high task demand. It occurs when capacity does not meet the task demands of the lowest cost strategy (global minimum). Then the next best strategy will be selected (local minimum) (Figure 2, 3).

- Compensation for Movement Objectives: relates to the emergence of different movement strategies due to a shift in the weighting of movement objectives.

In the ideal case, the movement-enhancing compensation is sufficient to meet the goal. If compensation no longer enables the execution of the task at hand, inability and mobility limitations will arise (Figure 2a). Capacity determines whether and which compensation strategies are available. Compensation and capacity are therefore overlapping and interacting. Individuals with greater capacity have more room to deploy effective compensation strategies. But compensation strategies can also be detrimental when they result in a habitual over- or underuse of physiological abilities. Elderly people can end up in a negative cycle (frailty cycle), which accelerates decline of capacity. This cycle is also prevalent in the young after traumatic incidents. In their case the compensation applied after a stressor, for example asymmetry in gait to unload the involved side, could permanently change their movement strategy. Such asymmetry could cause underuse of the involved side and overuse of the non-involved side, thereby putting capacity into decline in the long-term.

Within compensation we propose two forms or levels: selection and reorganization. Although these forms are not exclusive and can co-occur, it is important to recognize that multiple forms or levels of compensation exist (6):

- Compensation by selection: people can complete tasks using a variety of strategies to retain mobility including upper limb to lower limb compensations and postural changes. Compensation by selection is a variation in the planned movement trajectory. This compensation strategy is observable and can be expressed with the kinematics of the motion. Examples of compensation by selection are using the arms for standing up, using the handrail when climbing stairs, walking with a walking aid, widening the base of support in gait, or running with shorter step lengths. 
- Compensation by reorganization: this form of compensation engages the altered selection of muscle recruitment. This form of compensation co-occurs when compensation by selection is apparent. However, as there is a redundancy in the muscle architecture of the human body, compensation by reorganization can also occur without a change in trajectory. A possible need of reorganization in healthy ageing could be the relative difference in decline of muscle strength between muscle groups $(7,8)$. An example of this form of compensation is cocontraction, which is a strategy that can be executed to increase stability through changes only in muscle recruitment, rather than changes in kinematics. 


\section{Introducing CaReMoOC for ageing}

Quantifying the different components of CareMoOC requires various measurement techniques, and not all aspects can be measured with current technologies. Because not all parts can be measured directly, many studies have focused on an isolated variable and investigated how participants with high or low levels of these variables differ in their movements. The following section captures the current state of knowledge of age-related changes on all these variables in the context of biomechanics and motor control.

\subsection{Compensation for capacity}

Age-related decline in neuromusculoskeletal capacity can be divided separately into decline in neural, muscular and skeletal capacity. This section describes these age-related changes and considers how they can be measured.

\subsubsection{Neural capacity}

The neural system consists of the peripheral and central nervous system and the sensory feedback system, which can be divided into the visual, auditory, vestibular systems, and proprioception. These systems are critical for feedback control, which is an ongoing loop of acquisition and integration of sensory information that updates the current state and corrects posture accordingly in unperturbed and perturbed movements. These different feedback systems can decline independently (9) (Figure 4). However, systems can also compensate for each other.

\section{Proprioceptive acuity}

Proprioceptive feedback can come from muscle spindles, golgi tendon organs, and cutaneous and joint mechanoreceptors. The most common experiments to test the proprioceptive acuity in humans are 1) position tests, in which participants replicate a joint position, 2) motion sense tests, in which participants need to detect motion of any of the limbs, and 3) dynamic position tests, in which position and motion sense tests are combined (10). Joint position sense becomes more accurate through childhood and adolescence, peaks in young adulthood and then progressively deteriorates $(10,11)$. Ageing results in a decreased number and density of cutaneous and joint mechanoreceptors (12). This loss of sensation can be particularly impairing in the feet and hands, as the extremities are critical for movement and interaction with the environment. There is also an age-related reduction in the number of muscle spindles (13). As a consequence, the joint position and motor sense deteriorates with age 
(about 3\% per year between ages of 20 to 80 years for joint position) and affects motor tasks such as balance (11).

Visual acuity

Deterioration of vision due to ageing results in decrease of the visual acuity, decline in sensitivity of the visual field, decreased contrast sensitivity, and an increased dark adaptation threshold (14). The influence of visual feedback on complex motor task performance is typically determined by performing the task with eyes opened and closed or comparing cohorts with poor visual acuity (15). This result could indicate challenges with balance or a fear of falling in the poor visual acuity group. Fear of falling has been associated with poor visual acuity (16).

\section{Hearing acuity}

Although many adults retain good hearing as they age, hearing loss associated with ageing is common among elderly people (17). Hearing is influenced by genetics, environmental factors, such as exposure to loud noise, and intrinsic factors such as damage due to disease. Previous findings suggest that ambient sound and hearing have a significant influence on postural control and balance tested in a soundproof room (18).

\section{Vestibular acuity}

The vestibular system is responsible for an accurate perception of movement and self-orientation. Decline in the vestibular system results in balance deficits and can produce symptoms such as dizziness and a change in the control of head movements in standing up (19). Specialized equipment can evaluate vestibular acuity, yet the measurable effect of the deterioration of the vestibular sensory end organs remains elusive (20). Nonetheless, inquiring participants about any previous experienced balance losses or dizziness via a questionnaire could be beneficial for the interpretation of results in complex motor tasks.

\section{Nervous system}

Age-related changes in the nervous system have not been widely studied in complex motor tasks. In the central nervous system healthy ageing can result in gradual decline of cognitive processing, which can progress further through disease states such as dementia or step-wise following events such as a stroke. In either situation the ability of the brain to process and coordinate afferent information from neural circuits is impaired. This affects the ongoing loop of acquisition and integration of sensory information from the environment and movement planning. A simple test for cognitive function is the Clock Drawing Test, embedded in several cognitive assessments (21). 
The age-related structural changes in the peripheral and the spinal level of the neural system , which is when sensory and motor neurons and interneurons located in the spinal cord are involved, can be quantified by the reduction in nerve conduction velocity (NCV), response amplitude, and the increase in motor and sensor noise. The NCV and response amplitude can be studied by a nerve conduction study (22-24). Ageing affects afferent nerves (from the muscle to the spine) more than the efferent nerves $(25,26)$. However degeneration of efferent pathways is also evident, for example in the tibialis anterior muscle (27).

Both in young and elderly, motor and sensor noise are apparent due to cellular noise, unfused twitches, or cross-talk between nerves (28). With ageing, motor and sensor noise increases, which introduces variability in the execution of a trial-to-trial motion. An estimate of motor and sensor noise has previously been determined by combining a theoretical feedback control model with experimental data on kinematics of participants in standing balance on a platform, subjected to external perturbations (29). 

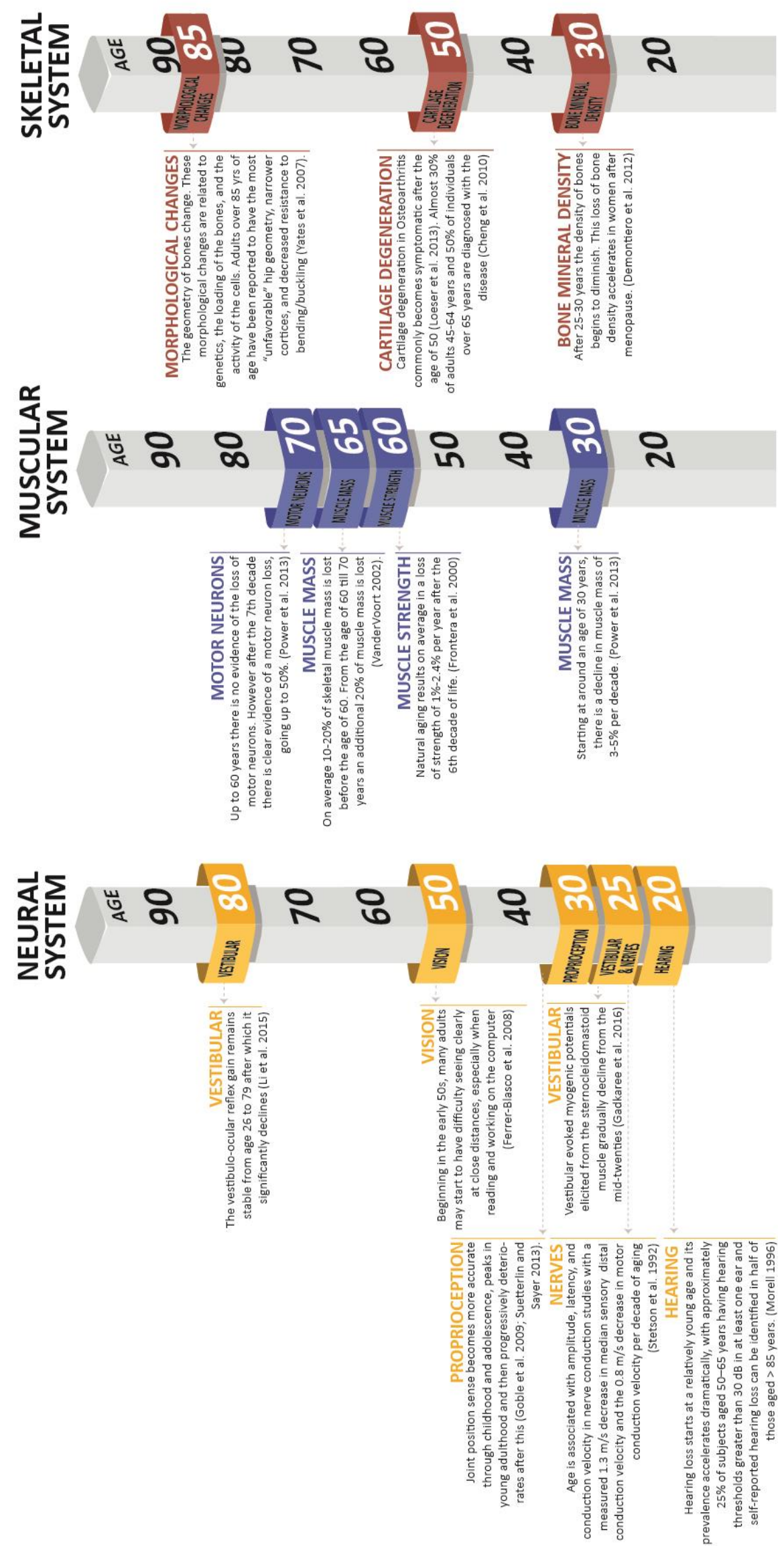

FIGURE 4: General onset of decline in the adult neural, muscular and skeletal system $(9-11,30)$. 


\subsubsection{Muscular capacity}

Muscle strength and power reduces with age due to a reduction in muscle mass, reduced number of fast-twitch muscle fibres, slower contraction speed, and slower excitation-contraction decoupling (31) (Figure 4). These reductions change the force-velocity relationship, resulting in shift of the normal curve to the left, due to decrease in contractile velocity at each relative load, and downward, due to loss of strength $(32,33)$; The loss of skeletal muscle mass is site-specific and likely associated with the pattern of muscle activations that occurs in daily life activities. During exercise, the muscles that are being used have up-regulated hormone receptors that bind androgen, a group of hormones with declining age-related concentrations associated with sarcopenia (34).

Relative muscle thickness of the quadriceps and trunk muscles gradually decreases with age, and in men only, arm, lower leg, and hamstrings muscle thickness reduces at old age (60-95 years) (8). Although the reduction in muscle thickness and associated muscle mass is associated with the decline in strength in older adults, muscle strength decline is more rapid than the loss of muscle mass, indicating that the quality of the muscle degrades (35). This rate of strength decline also varies between muscle groups (7).

The effect of reduced muscle strength on motor tasks is the most widely studied factor of reserve. It is difficult to determine whether elderly people actually lack muscle strength or if they have other deficits that prevent them from performing the task $(7,36,37)$. Different methods have been used to quantify muscle strength.

Handgrip strength has proven to be a useful tool to identify people at risk of mobility limitations (38) as a surrogate for overall muscle strength (39). Also, muscle strength reduction and its effect on task performance has been studied via exercise-induced muscle damage (40). Evaluating the maximum isometric (constant length contraction) or isokinetic (constant speed contraction) muscle strength (joint torque) of joints in relation to task performance can provide an indication of reserve in complex motor tasks. However, maximum voluntary joint torques are dependent on joint angle and angular velocity, due to the muscle force-length and force-velocity relationships. Directly predicting the (in)ability of complex motor tasks would therefore require a full angle-angular velocity torque profile of compensation strategies (41). Maximum voluntary contractions are also susceptible to large variability and are difficult to perform in impaired populations. Thus, isolated strength tests only partly reflect the actual strength available for a complex motor task. Also, studies that have added weight to change the load/capacity ratio in young adults cannot be directly applied to the change of load/capacity as observed with ageing. Muscle strength capacity with ageing declines at different rates 
between muscle groups (7). Therefore, such experimental protocols do not allow for compensation by reorganization, in which altered muscle recruitment is key.

Musculoskeletal modelling is an excellent tool to support the research in muscle weakness and movement strategies (42-44). Where experimental studies have measurement limitations, modelling can complement measurements and observations with movement simulations. For example, Bobbert et al. (2016) used a four-link two-dimensional rigid body model with nine muscle-tendon actuators to investigate successful sit-to-stand despite weakness of muscles. A common compensation strategy in standing up is pushing off on, for example, the armrests, which could be compensation for capacity or compensation for movement objectives. Their simulations indicated that a reduced muscle strength of up to $45 \%$ (all muscles) can still lead to a successful task completion without the use of arms. However, the cost of standing up (muscle activation squared as an indication of energy expenditure), was more than 2.5 times higher in the weakened simulations compared to the baseline model.

\subsubsection{Skeletal capacity}

The human skeletal system is constantly adapting (Figure 4). During development and ageing, bone shape responds to load and hormonal factors. Reshaping of the bone changes its functional abilities. Strength (stress to failure) is required to carry large loads, whereas toughness is required to absorb energy from an impact load. A proportional increase in tissue mineral content will generally yield stiffer (higher resistance to deformation) but more brittle bones (45). The effects combine so that with ageing, bones get stiffer, the cortices become thinner and toughness reduces, resulting in a greater likelihood of fracture. A common ageing disease is osteoporosis which results in more fragile bone and a greater risk of fragility fractures.

The strength of bone is usually expressed by the bone mineral density (BMD). However, compared to young adults with the same BMD, the risk of fracture is still higher in elderly people as bone shape and quality is not captured by BMD (46). Both cortical and trabecular bone becomes more brittle and weaker with age. Thus, it is the tissue-level properties in combination with the bone geometry that determine fracture risk. The most widely applied technique to measure bone mineral density is the dual energy $x$-ray absorptiometry (47).

Although the changed mechanics of bone at first sight do not directly translate to motor control of motor tasks, the consequences of change will affect movement. As the consequences of a fall are more severe (increased fracture risk), there is an increased fear of falling in the elderly, exacerbated by prior falls. This greater fear results in greater emphasis on stability as a movement objective in the motor control of movements (Figure 3). Furthermore, cartilage degeneration which is symptomatic in the disease Osteoarthritis $(\mathrm{OA})$ results in pain with loading, and therefore puts more emphasis on pain 
avoidance. For example, the most prominent compensation strategy of unilateral knee and hip OA patients in standing-up is asymmetry of movement (compensation by selection) (48-52). Knee OA patients bear additional weight on the unaffected side by leaning the trunk (lateral trunk lean) (48) or by using asymmetric arm movements (53). Pushing through the chair with the arm ipsilateral to the affected knee decreases the demand on lower limb extensors, while the arm on the contralateral side is used by swinging or pushing on the knee. Compensation by reorganisation is also apparent in knee OA patients in standing up where moderate knee OA patients try to maintain the same movement trajectory as healthy individuals, resulting in greater antagonist activation $(50,54,55)$. This greater activation most likely improves knee stability and provides relief from pain and discomfort.

Other biomechanical factors relevant for the modelling of human musculoskeletal systems, are changes in body mass distribution and reduced joint ranges of motion. The redistribution of body mass influences movement dynamics. A combination of muscle stiffness and skeletal changes results in a reduction of joint ranges of motion (ROM) with ageing. For example, the age-related reduced hip flexion range of motion approaches the maximal angle used in standing-up (56).

\subsection{Compensation for Movement Objectives}

Currently the biomechanical (modelling) community is undecided on which combination of movement objectives (cost-functions) best reproduces movement strategies that ageing humans choose for motor tasks $(43,57-59)$. The central nervous system may even apply different weighting for different phases of a complex task (59). Movement objectives feed into our feedforward planning, which is a control system to anticipate and compensate for potential disturbances. A major limiting factor in this research area is the lack of a reliable independent quantitative measures of key known movement objectives including energy, stability, and pain.

\subsubsection{Energy}

Biomechanical analyses frequently assume, based on sensible analysis, that humans are energyefficient and therefore minimization of energy is one of the major movement objectives. However, when performance is more important than energy efficiency (e.g., in sports), the relative weighting of this objective will change. In walking there is evidence that the weighting of movement objectives changes with ageing. Energy expenditure versus walking speed is nicely represented by a U-shape curve, where the minimum of the curve (minimal energy expenditure) matches the preferred walking speed in adults (60). In older adults, however, preferred walking speed declines and energy expenditure increases (61). First, the U-shape shifts up, meaning that walking effort increases overall. Secondly, as the U-shape does not shift to the left, but older adults do choose for a slower walking 
speed, this results in greater energy expenditure. Thus, older adults no longer walk at the minimum of the curve, but apparently have a shifted weighting in their movement objectives that results in a less economic movement pattern (61). Based on predictive neuromusculoskeletal modelling, the reason for this shift is postulated to be minimisation of muscle fatigue rather than the minimisation of metabolic cost of transport (62). Although many factors of age-related capacity decline have been included in this study, possible psychological reasons have not been incorporated, such as an increased emphasis on stability or pain. Therefore, comment cannot be made on the overall reason without taking into account all confounding factors.

A generally accepted measure of energy is estimation via measurement of energy consumption (VO2). Quantification of energy cost based on mechanics, and at the muscle level, however, is complex and literature is undecided on the most appropriate method (63). The most common used models for metabolic cost in biomechanical modelling are Umberger et al. (2003), Bhargava et al. (2004), Lichtwark and Wilson (2005), and Houdijk et al. (2006). The most applied experimental measures to analyse energy objectives are relative effort measures in which the maximum torque measured in the motor task is normalized to the maximum voluntary isometric torque $(36,37)$. Alternatively, measured torques can be normalized to a theoretical maximum joint torque, based on joint angle and angular velocity (41).

\subsubsection{Pain avoidance}

The presence of pain substantially affects movement strategies. Previously, pain has been quantified by the WOMAC pain questionnaire, or the Visual Analogue Scale (VAS). Psychological assessments are scarcely reported in experimental biomechanics literature (68) (68)(68), despite standardized questionnaire tools that are available to assess pain $(69,70)$. Incorporating a measure of pain is important, especially in the elderly, as pain becomes more prevalent with ageing. Pain avoidance can also lead to a long-term compensation, long after the pain is mitigated or eliminated (71). Former stressors or pain in participants might therefore still influence current movement strategies, which need to be accounted for when analysing movement strategies.

\subsubsection{Stability}

More severe consequences of injury and higher likelihood of falls increase the fear of falling in elderly people. A history of falls, lower levels of overall self-esteem, as well as flexibility and reduced knee strength are all predictors for fear of falling (39). Compensation due to fear of falling is a feedforward adjustment to reduce the likelihood of balance loss, which is apparent in elderly, fallers (elderly people with a history of falling), and in young adults in uncertain environments (72). For example, in standing 
up, adults with a history of falls show different movement strategies compared to non-fallers regardless of their capacity, showing compensation for movement objectives $(73,74)$.

\subsubsection{Speed}

Speed instructions trigger compensation of selection and reorganization and thus setting and reporting on the speed instructions in experimental protocols is important. Movement velocity is a task-driven variable. Elderly people can reduce the duration of their movements when asked to move as fast as possible and are therefore not bound to their initial self-selected speed (75).

\subsection{Compensation in experimental set-ups}

Experimental design plays an important part in facilitating or constraining available compensation strategies. The design of an experiment is a trade-off between 1) standardization of the protocol to improve repeatability, robustness, and comparability and 2) replication of daily practice to allow for clinical translation. Notwithstanding the differences between experiments, most prior studies on agerelated mobility limitations have typically used standardized experimental protocols, restricting compensation in their protocols. For example, most studies on sit-to-stand do not permit the participants to compensate by using their arms. This restriction poorly reflects the importance of arms in daily life, as more than half of the healthy elderly population is unable to stand up without the use of arms, and more than half of all adults prefer to use their arms when standing up $(76,77)$. Such restricted experimental protocols facilitate comparison between groups and studies, but also limit their translation to characterising mobility of the elderly in their homes, communities, and clinic.

The aim of this article is not to argue that all elements of capacity should be incorporated in experimental protocols. However, being aware of the neglected parts of age-related changes may improve interpretation of experimental results. Furthermore, the areas that require further knowledge to either be implemented into biomechanics and motor control (for example a realistic energetics model) and the research areas that require more research (for example determination of reserve in motor tasks) have been outlined here. 


\section{Conclusions}

CaReMoOC is a framework incorporating neuromuscular capacity (accumulation of NMSK resources over the lifespan), reserve (task-specific difference between capacity and task demand), movement objectives (considerations made to plan a movement), and compensation (use of NMSK resources to respond to the task demand). With healthy ageing the NMSK capacity declines. This decline is apparent in the neural, muscular, and skeletal systems and each have their effect on the execution of complex motor tasks. For a specific task, humans have NMSK reserve, so that, if NMSK capacity reduces, the task is not necessarily impaired. Moreover, humans have multiple compensation strategies to meet the task goal. These compensation strategies can be based on changed movement trajectories (selection) or enhanced muscle recruitment (reorganization). There are two cases of compensation; Compensation for capacity, when capacity does not meet the task demands, or Compensation for Movement Objectives, due to psychological reasons. Knowledge of reserve and compensation are of importance to maintain mobility in elderly people since compensation strategies that are beneficial in the short-term, may become detrimental for the capacity in the long-term. To understand the interrelation between decline in the variables within capacity and their effect on compensation strategies will benefit prevention of mobility impairments and support clinicians in their rehabilitation practice.

\section{List of Abbreviations}

\begin{tabular}{|l|l|}
\hline BMD & Bone Mineral Density \\
\hline CaReMoOC & $\begin{array}{l}\text { Referring to the framework of Capacity, Reserve, Movement Objectives, and } \\
\text { Compensation }\end{array}$ \\
\hline NMSK & Neuromusculoskeletal \\
\hline OA & Osteoarthritis \\
\hline ROM & Range of Motion \\
\hline VAS & Visual Analogue Scale \\
\hline
\end{tabular}




\section{References}

1. UN. United Nations. 2019.

2. Oseid S. Physical work capacity in puberty. Physiological capacity, sex differences and possibilities of influence. Tidsskr den Nor laegeforening Tidsskr Prakt Med ny raekke. 1973;93(14):1007-11.

3. Bull AMJ, Cleather D, Southgate D. Musucloskeletal reserve: A tool to quantify frailty in ageing. In: Bioengineering 08 London. 2008. p. 49.

4. Nygård C-H, Luopajärvi T, Cedercreutz G, Ilmarinen J. Musculoskeletal capacity of employees aged 44 to 58 years in physical, mental and mixed types of work. Eur J Appl Physiol Occup Physiol. 1987;56(5):555-61.

5. Papa E, Cappozzo A. Sit-to-stand motor strategies investigated in able-bodied young and elderly subjects. J Biomech. 2000;33(9):1113-22.

6. Cabeza R, Albert M, Belleville S, Craik FIM, Duarte A, Grady CL, et al. Maintenance, reserve and compensation: the cognitive neuroscience of healthy ageing. Nat Rev Neurosci. 2018;1.

7. Gross MM, Stevenson PJ, Charette SL, Pyka G, Marcus R. Effect of muscle strength and movement speed on the biomechanics of rising from a chair in healthy elderly and young women. Gait Posture. 1998;8:175-85.

8. Abe T, Sakamaki M, Yasuda T, Bemben MG, Kondo M, Kawakami Y, et al. Age-related, sitespecific muscle loss in 1507 Japanese men and women aged 20 to 95 years. J Sports Sci Med. 2011;10(1):145.

9. Gadkaree SK, Sun DQ, Li C, Lin FR, Ferrucci L, Simonsick EM, et al. Does sensory function decline independently or concomitantly with age? Data from the Baltimore longitudinal study of aging. J Aging Res. 2016;2016.

10. Suetterlin KJ, Sayer AA. Proprioception: where are we now? A commentary on clinical assessment, changes across the life course, functional implications and future interventions. Age Ageing. 2013;43(3):313-8.

11. Goble DJ, Coxon JP, Wenderoth N, Van Impe A, Swinnen SP. Proprioceptive sensibility in the elderly: degeneration, functional consequences and plastic-adaptive processes. Neurosci Biobehav Rev. 2009;33(3):271-8.

12. Lee NK, Kwon YH, Son SM, Nam SH, Kim JS. The effects of aging on visuomotor coordination 
and proprioceptive function in the upper limb. J Phys Ther Sci. 2013;25(5):627-9.

13. Kararizou E, Manta P, Kalfakis N, Vassilopoulos D. Morphometric study of the human muscle spindle. Anal Quant Cytol Histol. 2005;27(1):1-4.

14. Salvi SM, Akhtar S, Currie Z. Ageing changes in the eye. Postgrad Med J. 2006;82(971):581-7.

15. Shin S-S, An D-H, Yoo W-G. Comparison of Foot Pressure and Center of Force During Sit-toStand and Stand-to-Sit Movements in Older Adults With Good and Poor Visual Acuity. Top Geriatr Rehabil. 2018;34(1):82-6.

16. Aartolahti E, Häkkinen A, Lönnroos E, Kautiainen H, Sulkava R, Hartikainen S. Relationship between functional vision and balance and mobility performance in community-dwelling older adults. Aging Clin Exp Res. 2013;25(5):545-52.

17. Liu XZ, Yan D. Ageing and hearing loss. J Pathol A J Pathol Soc Gt Britain Irel. 2007;211(2):18897.

18. Kanegaonkar RG, Amin K, Clarke M. The contribution of hearing to normal balance. J Laryngol Otol. 2012;126(10):984-8.

19. Tsutsumi T, Nozawa M, Inaoka H, Fukuoka Y, Ishida A, Kitamura K. Time course analysis of angular control of the body and head while rising from a chair. Acta Otolaryngol. 2004;124(7):798-802.

20. Zalewski CK. Aging of the human vestibular system. In: Seminars in hearing. Thieme Medical Publishers; 2015. p. 175-96.

21. Woodford HJ, George J. Cognitive assessment in the elderly: a review of clinical methods. QJM An Int J Med. 2007;100(8):469-84.

22. Palmieri RM, Ingersoll CD, Hoffman MA. The Hoffmann reflex: methodologic considerations and applications for use in sports medicine and athletic training research. J Athl Train. 2004;39(3):268.

23. Frijns CJM, Laman DM, Van Duijn MAJ, Van Duijn H. Normal values of patellar and ankle tendon reflex latencies. Clin Neurol Neurosurg. 1997;99(1):31-6.

24. Sadeghi S, Ghavanini M, Ashraf A, Jafari P. Effects of age and leg length upon central loop of the gastrocnemius-soleus H-reflex latency. BMC Neurol. 2004;4(1):11.

25. Papegaaij S, Taube W, Baudry S, Otten E, Hortobágyi T. Aging causes a reorganization of 
cortical and spinal control of posture. Front Aging Neurosci. 2014;6:28.

26. Scaglioni G, Narici M V, Maffiuletti NA, Pensini M, Martin A. Effect of ageing on the electrical and mechanical properties of human soleus motor units activated by the $\mathrm{H}$ reflex and $\mathrm{M}$ wave. J Physiol. 2003;548(2):649-61.

27. McNeil CJ, Doherty TJ, Stashuk DW, Rice CL. Motor unit number estimates in the tibialis anterior muscle of young, old, and very old men. Muscle Nerve Off J Am Assoc Electrodiagn Med. 2005;31(4):461-7.

28. Faisal AA, Selen LPJ, Wolpert DM. Noise in the nervous system. Nat Rev Neurosci. 2008;9(4):292.

29. Van Der Kooij H, Peterka RJ. Non-linear stimulus-response behavior of the human stance control system is predicted by optimization of a system with sensory and motor noise. J Comput Neurosci. 2011;30(3):759-78.

30. Cheng YJ, Hootman JM, Murphy LB, Langmaid GA, Helmich CG. Prevalence of doctordiagnosed arthritis and arthritis-attributable activity limitation-United States, 2007-2009. Morb Mortal Wkly Rep. 2010;59(39):1261-5.

31. Mitchell WK, Williams J, Atherton P, Larvin M, Lund J, Narici M. Sarcopenia, dynapenia, and the impact of advancing age on human skeletal muscle size and strength; a quantitative review. Front Physiol. 2012;3.

32. Power GA, Dalton BH, Rice CL. Human neuromuscular structure and function in old age: a brief review. J Sport Heal Sci. 2013;2(4):215-26.

33. Vandervoort AA. Aging of the human neuromuscular system. Muscle Nerve Off J Am Assoc Electrodiagn Med. 2002;25(1):17-25.

34. Morley JE. Hormones and the aging process. J Am Geriatr Soc. 2003;51(7s):S333-7.

35. Goodpaster BH, Park SW, Harris TB, Kritchevsky SB, Nevitt M, Schwartz A V, et al. The loss of skeletal muscle strength, mass, and quality in older adults: the health, aging and body composition study. Journals Gerontol Ser A Biol Sci Med Sci. 2006;61(10):1059-64.

36. Hughes MA, Schenkman ML. Chair rise strategy in the functionally impaired elderly. J Rehabil Res Dev. 1996;33(4):409-12.

37. Kotake T, Miura T, Kajiwara T. An Analysis of Sit-to-Stand Movements. 1993;74(October):1095-9. 
38. Sallinen J, Stenholm S, Rantanen T, Heliövaara M, Sainio P, Koskinen S. Hand-grip strength cut points to screen older persons at risk for mobility limitation. J Am Geriatr Soc. 2010;58(9):1721-6.

39. Sales $\mathrm{M}$, Levinger $\mathrm{P}$, Polman $\mathrm{R}$. Relationships between self perceptions and physical activity behaviour, fear of falling, and physical function among older adults. Eur Rev aging Phys Act. 2017;14(1):17.

40. Spyropoulos G, Tsatalas T, Tsaopoulos DE, Sideris V, Giakas G. Biomechanics of sit-to-stand transition after muscle damage. Gait Posture [Internet]. 2013;38(1):62-7. Available from: http://dx.doi.org/10.1016/j.gaitpost.2012.10.013

41. Anderson DE, Madigan ML, Nussbaum MA. Maximum voluntary joint torque as a function of joint angle and angular velocity: model development and application to the lower limb. J Biomech. 2007;40(14):3105-13.

42. Bajelan S, Azghani MR. Musculoskeletal modeling and simulation of three various Sit-to-Stand strategies : An evaluation of the biomechanical effects of the chair-rise strategy modification. $2014 ; 22: 627-44$.

43. Bobbert MF, Houdijk H, De Koning JJ, De Groot G. From a one-legged vertical jump to the speed-skating push-off: A simulation study. J Appl Biomech [Internet]. 2002;18(1):28-45. Available from: https://www.scopus.com/inward/record.uri?eid=2-s2.00036171210\&partnerID=40\&md5=7011616 dbfbe1a343d5fcbd2ef0dfd62

44. Caruthers EJ, Thompson JA, Chaudhari AMW, Schmitt LC, Best TM, Saul KR, et al. Muscle forces and their contributions to vertical and horizontal acceleration of the center of mass during sitto-stand transfer in young, healthy adults. J Appl Biomech. 2016;32(5):487-503.

45. Boskey AL, Coleman R. Aging and bone. J Dent Res. 2010;89(12):1333-48.

46. Kanis JA. Diagnosis of osteoporosis and assessment of fracture risk. Lancet. 2002;359(9321):1929-36.

47. Shewale P, Aglawe A, Ambrose A, Patta R, Choudhari P. Techniques used for Bone Density Measurement. Int J Comput Appl. 2017;178(3):20-3.

48. Turcot K, Armand S, Fritschy D, Hoffmeyer P, Suvà D. Sit-to-stand alterations in advanced knee osteoarthritis. Gait Posture. 2012;36(1):68-72.

49. Naili E, Broström EW, Gutierrez-farewik EM, Schwartz MH. The centre of mass trajectory is a 
sensitive and responsive measure of functional compensations in individuals with knee osteoarthritis performing the five times sit-to-stand test. Gait Posture. 2018;62:140-5.

50. Davidson BS, Judd DL, Thomas AC, Mizner RL, Eckhoff DG, Stevens-Lapsley JE. Muscle activation and coactivation during five-time-sit-to-stand movement in patients undergoing total knee arthroplasty. J Electromyogr Kinesiol [Internet]. 2013;23(6):1485-93. Available from: http://dx.doi.org/10.1016/j.jelekin.2013.06.008

51. Eitzen I, Fernandes L, Nordsletten L, Snyder-Mackler L, Risberg MA. Weight-bearing asymmetries during Sit-To-Stand in patients with mild-to-moderate hip osteoarthritis. Gait Posture [Internet]. 2014;39(2):683-8. Available from: http://dx.doi.org/10.1016/j.gaitpost.2013.09.010

52. Lamontagne $M$, Beaulieu ML, Varin D, Beaulé PE. Lower-limb joint mechanics after total hip arthroplasty during sitting and standing tasks. J Orthop Res. 2012;30(10):1611-7.

53. Martin J, Garn A, Studies S, Rouge B. Note : This article will be published in a forthcoming issue of The Sport Psychologist. This article appears here in its accepted, peer-reviewed form, as it was provided by the submitting author. It has not been copy edited, proofed, or formatted by. 2013;

54. Patsika G, Kellis E, Amiridis IG. Neuromuscular efficiency during sit to stand movement in women with knee osteoarthritis. J Electromyogr Kinesiol [Internet]. 2011;21(5):689-94. Available from: http://dx.doi.org/10.1016/j.jelekin.2011.05.006

55. Bouchouras G, Patsika G, Hatzitaki V, Kellis E. Kinematics and knee muscle activation during sitto-stand movement in women with knee osteoarthritis. Clin Biomech. 2015;30(6):599-607.

56. Fotoohabadi MR, Tully EA, Galea MP. Kinematics of Rising From a Chair : Image-Based Analysis of the Sagittal Elderly People Who Are Healthy. Phys Ther. 2010;90(4):561-71.

57. Mughal AM, Perviaz S, Iqbal K. LMI based physiological cost optimization for biomechanical STS transfer. Conf Proc - IEEE Int Conf Syst Man Cybern. 2011;1508-13.

58. Mughal A, Iqbal K. 3D Bipedal Model With Holonomic Constraints for the Decoupled Optimal Controller Design of the Biomechanical Sit-to-Stand Maneuver. J Biomech Eng. 2010;132:1-9.

59. Sadeghi M, Emadi M. Trajectory of human movement during sit to stand : a new modeling approach based on movement decomposition and multi - phase cost function. Exp Brain Res. 2013;229:221-34. 
60. Pearce ME, Cunningham DA, Donner AP, Rechnitzer PA, Fullerton GM, Howard JH. Energy cost of treadmill and floor walking at self-selected paces. Eur J Appl Physiol Occup Physiol. $1983 ; 52(1): 115-9$.

61. Malatesta D, Simar D, Dauvilliers Y, Candau R, Borrani F, Préfaut C, et al. Energy cost of walking and gait instability in healthy 65-and 80-yr-olds. J Appl Physiol. 2003;95(6):2248-56.

62. Song S, Geyer H. Predictive neuromechanical simulations indicate why walking performance declines with ageing. J Physiol. 2018;596(7):1199-210.

63. Van Der Kruk E, Van Der Helm FCT, Veeger HEJ, Schwab AL. Power in Sports: a literature review on the application, assumptions, and terminology of mechanical power in sport research. Artic J Biomech [Internet]. 2018; Available from: https://doi.org/10.1016/j.jbiomech.2018.08.031

64. Umberger BR, Gerritsen KGM, Martin PE. A model of human muscle energy expenditure. Comput Methods Biomech Biomed Engin. 2003;6(2):99-111.

65. Bhargava LJ, Pandy MG, Anderson FC. A phenomenological model for estimating metabolic energy consumption in muscle contraction. J Biomech. 2004;37(1):81-8.

66. Lichtwark GA, Wilson AM. In vivo mechanical properties of the human Achilles tendon during one-legged hopping. J Exp Biol. 2005;208(24):4715-25.

67. Houdijk H, Bobbert MF, De Haan A. Evaluation of a Hill based muscle model for the energy cost and efficiency of muscular contraction. J Biomech. 2006;39(3):536-43.

68. Frykberg GE, Häger CK. Movement analysis of sit-to-stand - research informing clinical practice. Phys Ther Rev [Internet]. 2015;20(3):156-67. Available from: http://www.tandfonline.com/doi/full/10.1179/1743288X15Y.0000000005

69. Yardley L, Beyer N, Hauer K, Kempen G, Piot-Ziegler C, Todd C. Development and initial validation of the Falls Efficacy Scale-International (FES-I). Age Ageing. 2005;34:614-9.

70. Linton SJ, Boersma K. Early identification of patients at risk of developing a persistent back problem: the predictive validity of the Örebro Musculoskeletal Pain Questionnaire. Clin J Pain. $2003 ; 19(2): 80-6$.

71. Farquhar SJ, Reisman DS, Snyder-Mackler L. Persistence of Altered Movement Patterns During a Sit-to-Stand Task 1 Year Following Unilateral Total Knee Arthroplasty. Phys Ther [Internet]. 2008;88(5):567-79. Available from: https://academic.oup.com/ptj/article/2742396/Persistence 
72. Pavol MJ, Pai YC. Feedforward adaptations are used to compensate for a potential loss of balance. Exp Brain Res. 2002;145(4):528-38.

73. Chorin F, Cornu C, Beaune B, Frère J, Rahmani A. Sit to stand in elderly fallers vs non-fallers: new insights from force platform and electromyography data. Aging Clin Exp Res. 2016;28(5):871-9.

74. Chen T, Chang CC, Chou LS. Sagittal plane center of mass movement strategy and joint kinetics during sit-to-walk in elderly fallers. Clin Biomech [Internet]. 2013;28(7):807-12. Available from: http://dx.doi.org/10.1016/j.clinbiomech.2013.07.002

75. Vander Linden DW, Brunt D, McCulloch MU. Variant and invariant characteristics of the sit-tostand task in healthy elderly adults. Arch Phys Med Rehabil. 1994;75(6):653-60.

76. Komaris D-S, Govind C, Murphy A, Ewen A, Riches P. Identification of movement strategies during the sit-to-walk movement in patients with knee osteoarthritis. J Appl Biomech. 2018;34(2):96-103.

77. Mazzà C, Benvenuti F, Bimbi C, Stanhope SJ. Association Between Subject Functional Status, Seat Height, and Movement Strategy in Sit-to-Stand Performance. J Am Geriatr Soc. 2004;52(10):1750-4. 